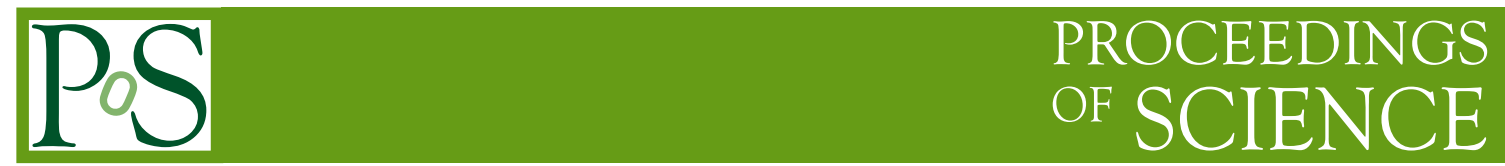

\title{
Searches for exotic baryonic states at $\mathrm{LHCb}$
}

\author{
Paolo Gandini, on behalf of the LHCb collaboration* \\ Istituto Nazionale di Fisica Nucleare \\ E-mail: paolo.gandini@cern.ch
}

\begin{abstract}
With the discovery of two pentaquark candidates at LHCb, a new field of baryon spectroscopy has been opened. The unique data samples on beauty baryon decays collected at the LHC during its runs I and II provide opportunities to further study the spectrum of exotic heavy baryons. This presentation will highlight recent progress on establishing new baryonic multiparticle decay modes of beauty hadrons and will summarize the status of the pentaquark searches.
\end{abstract}

The 39th International Conference on High Energy Physics (ICHEP2018)

4-11 July, 2018

Seoul, Korea

${ }^{*}$ Speaker. 


\section{Introduction}

The $\mathrm{LHCb}$ detector has proven to be an exceptional laboratory for heavy-hadron physics. With its unprecedented statistics, high cross sections for heavy quark production, excellent Particle Identification (PID) and trigger capabilities, LHCb provides a unique environment for baryon spectroscopy. The first pentaquark observation in 2015 [1] opened a new field for exotics in the baryon sector. Several final states have been investigated so far exhibiting evidence for pentaquark candidates, with further modes becoming accessible experimentally the more luminosity is collected by the experiment. In these proceedings, I will highlight the recent progress on baryonic multiparticle modes made by the LHCb collaboration.

\section{Search for weakly decaying b-favoured pentaquarks [2]}

The observation of charmonium pentaquark states with quark content $c \bar{c} u u d$ by the LHCb [1] collaboration raised many questions about the internal structure of these structures. This work presents the search for pentaquark states containing a single $b$ (anti)quark decaying weakly into four specific final states: $J / \Psi K^{+} \pi^{-} p, J / \Psi K^{-} \pi^{-} p, J / \Psi K^{-} \pi^{+} p$, and $J / \Psi \phi(1020) p$. These possible pentaquark states $\bar{b} d u u d$, bu $u d d, b \bar{d} u u d$ and $\bar{b}$ suud are labeled $P_{B^{0} p}^{+}, P_{\Lambda_{b}^{0} \pi^{-}}^{-}, P_{\Lambda_{b}^{0} \pi^{+}}^{+}$and $P_{B_{s}^{0}}^{+}$, respectively, where the subscript indicates the final states the pentaquark would predominantly decay into if it had sufficient mass to decay strongly into those states. The data sample used for the analysis corresponds to an integrated luminosity of $3.0 \mathrm{fb}^{-1}$ in 7 and $8 \mathrm{TeV} p p$ collisions. Weak decays exhibit appealing experimental features, e.g. detached vertices that could be used in the selection to further reduce prompt backgrounds, while no existing searches for weakly decaying pentaquarks containing a $b$ (anti)quark have been published to date. The Feynman diagrams for the decay of the $P_{B^{0} p}^{+}$and $P_{B_{s}^{0} p}^{+}$states are shown in Fig. 1. The corresponding diagrams for the decay of $P_{\Lambda_{b}^{0} \pi^{-}}^{-}$and $P_{\Lambda_{b}^{0} \pi^{+}}^{+}$are similar to that shown in Fig. 1(a), with the decay of the state being driven by the $b \rightarrow c \bar{c} s$ transition. Signal candidates are selected with a multivariate classifier trained using simulated events. Signals are not observed and upper limits (UL) at $90 \%$ confidence level are set on the product of the production cross section times branching fraction with respect to the production and decay of the $\Lambda_{b}^{0}$ baryon in the mode $J / \Psi K^{-} p$. ULs are set in each $P_{B}$ candidate mass interval

(a)

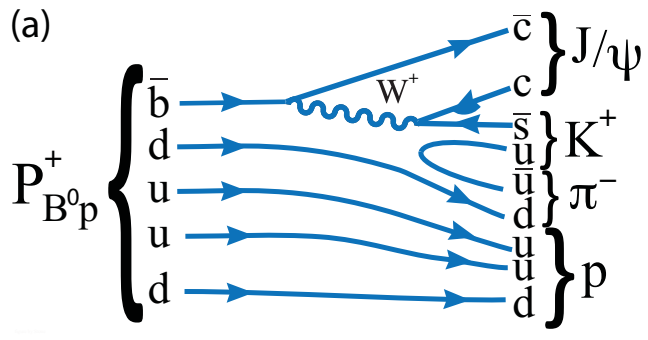

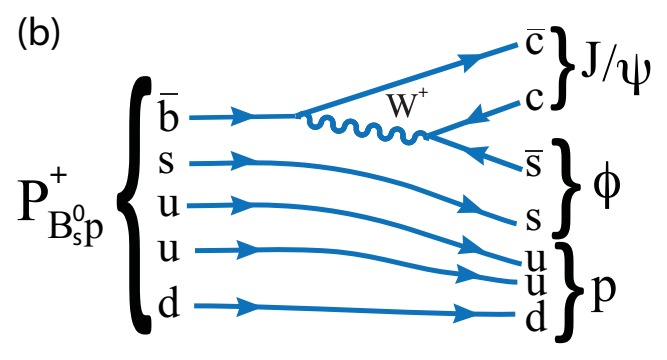

Figure 1: Leading-order diagrams for pentaquark decay modes into (a) $J / \Psi K^{+} \pi^{-} p$ or (b) $J / \Psi \phi p$ final states. 

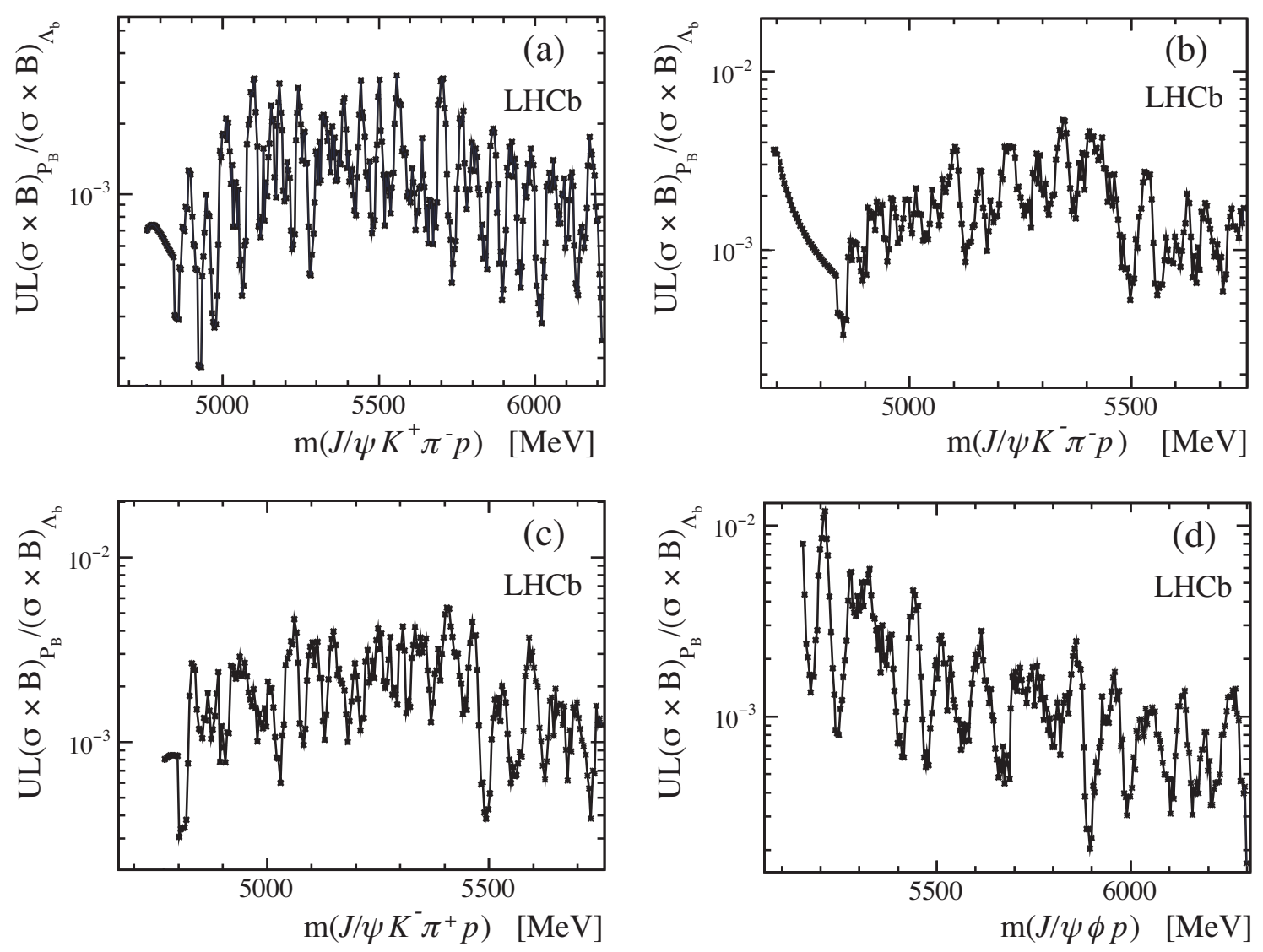

Figure 2: Upper limits on $R$ at $90 \% \mathrm{CL}$ for (a) $J / \Psi K^{+} \pi^{-} p$, (b) $J / \Psi K^{-} \pi^{-} p$, (c) $J / \Psi K^{-} \pi^{+} p$, and (d) $J / \Psi \phi p$ final states.

on the ratio $R$ :

$$
R=\frac{\sigma\left(p p \rightarrow P_{B} X\right) \cdot \mathscr{B}\left(P_{B} \rightarrow J / \Psi X\right)}{\sigma\left(p p \rightarrow \Lambda_{b}^{0} X\right) \cdot \mathscr{B}\left(\Lambda_{b}^{0} \rightarrow J / \Psi K^{-} p\right)}
$$

The numerical values are shown in Fig. 2 for the different hypothesized masses of the pentaquark states.

\section{Observation of the decay $\Lambda_{b}^{0} \rightarrow \Psi(2 S) p \pi^{-}$[3]}

The $\Lambda_{b}^{0}$ decays to the excited charmonium state $\Psi(2 S)$ have been observed by the ATLAS and LHCb collaborations [4, 5]. The Cabibbo-suppressed analogue of the latter decay, $\Lambda_{b}^{0} \rightarrow \Psi(2 S) p \pi^{-}$ is of particular interest because of possible contributions from exotic states in both the $\Psi(2 S) p$ system, similar to the $\mathrm{P}_{c}(4380)^{+}$and $\mathrm{P}_{c}(4450)^{+}$pentaquark states, and in the $\Psi(2 S) \pi^{-}$system, analogous to the charged charmonium-like state $Z_{c}(4430)^{-}$. Depending on the nature of a proposed exotic state, its coupling with the $\Psi(2 S)$ meson can be larger than the $J / \Psi$ meson. This paper reports the first observation of the decay $\Lambda_{b}^{0} \rightarrow \Psi(2 S) p \pi^{-}$, using a data sample of $p p$ collisions corresponding to $1.0,2.0$ and $1.9 \mathrm{fb}^{-1}$ of integrated luminosity at $\sqrt{s}=7,8$ and $13 \mathrm{TeV}$, respectively. The mass distributions for the selected $\Lambda_{b}^{0} \rightarrow \Psi(2 S) p \pi^{-}$and $\Lambda_{b}^{0} \rightarrow \Psi(2 S) p K^{-}$candidates are shown in Fig. 3. The signal yields are determined to be $121 \pm 13$ and $806 \pm 29$, respectively. 

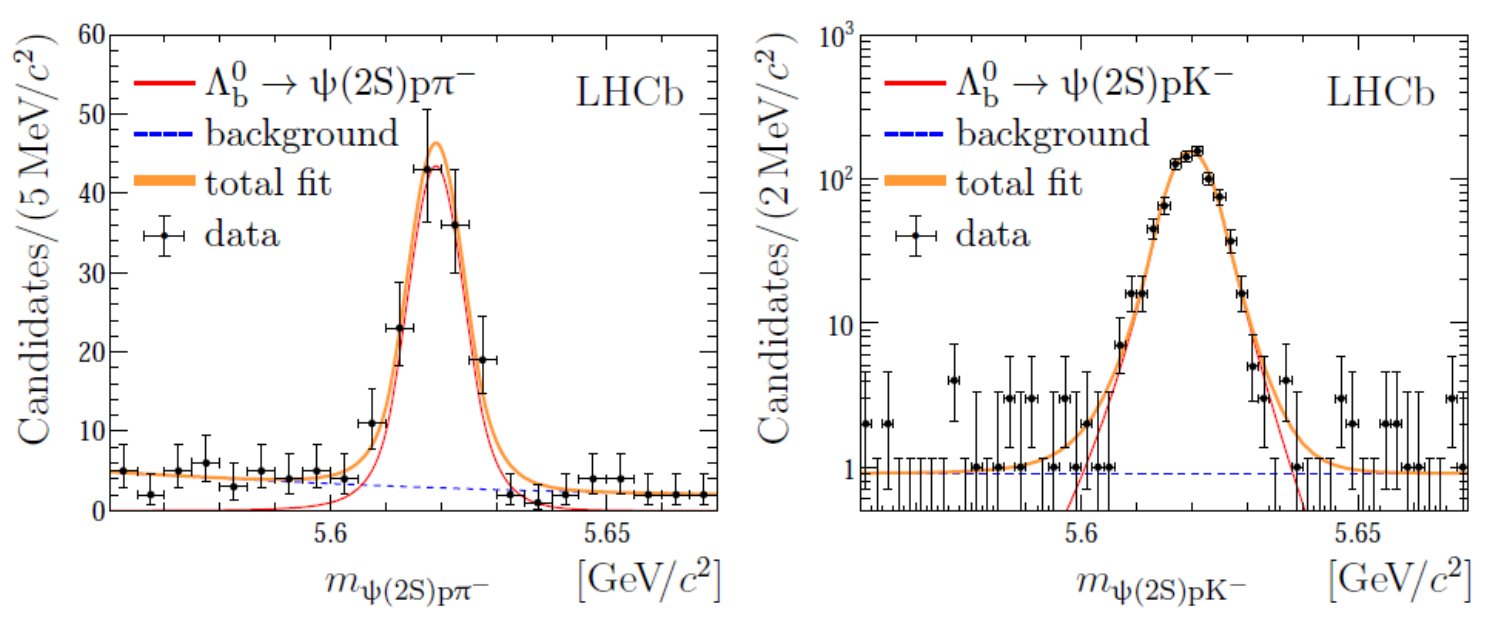

Figure 3: Mass distributions of the (left) $\Lambda_{b}^{0} \rightarrow \Psi(2 S) p \pi^{-}$and (right) $\Lambda_{b}^{0} \rightarrow \Psi(2 S) p K^{-}$candidates.

The efficiency is derived as the product of the geometric acceptance and the detection, reconstruction, selection and trigger efficiencies, which are either estimated by a data-driven approach or taken from simulation. The branching fraction is measured relative to that of the Cabibbo-favoured decay $\Lambda_{b}^{0} \rightarrow \Psi(2 S) p K^{-}$

$$
R_{\pi / K} \equiv \frac{\mathscr{B}\left(\Lambda_{b}^{0} \rightarrow \Psi(2 S) p \pi^{-}\right)}{\mathscr{B}\left(\Lambda_{b}^{0} \rightarrow \Psi(2 S) p K^{-}\right)}=(11.4 \pm 1.3 \pm 0.2)
$$

where the first uncertainty is statistical and the second is systematic. The $\Psi(2 S) p$ and $\Psi(2 S) p$ mass spectra are investigated and no evidence for contributions from exotic states is found. With a larger data sample a detailed amplitude analysis of this decay could be performed, making it possible to search for small contributions from exotic states.

\section{References}

[1] R. Aaij et al., Observation of $J / \Psi p$ resonances consistent with pentaquark states in $\Lambda_{b}^{0} \rightarrow J / \Psi K^{-} p$ decays, PRL 115 (2015), 072001.

[2] R. Aaij et al., Search for weakly decaying b-flavored pentaquarks, PRD 97 (2018), 032010.

[3] R. Aaij et al., Observation of the decay $\Lambda_{b}^{0} \rightarrow \Psi(2 S) p \pi^{-}, J H E P 1808$ (2018), 131.

[4] G. Aad et al., Measurement of the branching ratio $\Gamma\left(\Lambda_{b}^{0} \rightarrow \Psi(2 S) \Lambda^{0}\right) / \Gamma\left(\Lambda_{b}^{0} \rightarrow J / \Psi \Lambda^{0}\right)$ with the ATLAS detector, PLB 751 (2015), 63.

[5] R. Aaij et al., Observation of $\Lambda_{b}^{0} \rightarrow \Psi(2 S) p K^{-}$and $\Lambda_{b}^{0} \rightarrow J / \Psi \pi^{+} \pi^{-} p K^{-}$decays and a measurement of the $\Lambda_{b}^{0}$ baryon mass, JHEP $\mathbf{0 5}$ (2016), 132. 